5. Зефиров Ю.В., Зоркий П.М. // Успехи химии. 1989. № 5. Т. 58. С. 713-746; 1995. № 5. T. 64. С. 446-460.

6. Зоркий П.М., Зоркая О.Н. // Журн. структур. химии. 2000. № 5. Т. 41. C. 1054-1066.

7. Малеев А.В., Седов Б.Б., Житков И.К., Рау В.Г. // Журн. структур. химии. 2007. № 1. Т. 48. С. 148.

8. Shishkin O.V., Dyakonenko V.V., Maleev A.V., Schollmeyer D., Vysotsky M.O. // CrystEngComm. 2011. T. 13. P. 800.

9. Shishkin O.V., Dyakonenko V.V., Maleev A.V. // CrystEngComm. 2012. T. 14. P. 1795.

10. Shishkin O.V., Zubatyuk R.I., Maleev A.V., Boese R. // Structural Chemistry. 2014. T. 25. № 5. Р. 1547.

11. Грибов Л.А. // Журн. структур. химии. 2005. Т. 46. № 2. С. 311.

12. Williams D.E., Houpt D.J. // Acta Crystallographica. 1986. B 42. P. 286.

\title{
ОЦЕНКА ЧИСЛА p2-РАЗБИЕНИЙ ПЛОСКОСТИ НА ПОЛИГЕКСЫ
} ЗАДАННОЙ ПЛОЩАДИ

https://doi.org/10.31241/MIEN.2018.14.11

Шутов А.В. ${ }^{1}$, Коломейкина Е.В. ${ }^{2}$

${ }^{1}$ Владимирский государственный университет, Владимир, a1981@mail.ru

${ }^{2}$ МГТУ им. Н.Э. Баумана, Москва, pihta2@rambler.ru

\section{Аннотация}

В работе рассматривается задача о числе $p 2$-разбиений плоскости на полигексы заданной площади. То есть таких разбиений, при которых любую фигуру разбиения можно перевести в любую другую фигуру этого разбиения параллельным переносом либо центральной симметрией, причем преобразование переводит все разбиение в себя. Пусть $t(n)$ - число $p 2$-разбиений плоскости на полигексы площади $n$. Доказано, что справедливо неравенство $C_{1} \cdot 2^{n} \leq t(n) \leq C_{2} \cdot n^{5} \cdot 3,42^{n}$. При доказательстве нижней оценки использована явная конструкция, позволяющая построить требуемое число $p 2$-разбиений плоскости. Доказательство верхней оценки основано на критерии Конвея существования $p 2$-разбиений плоскости и на теории самонепересекающихся блужданий на гексагональной решетке.

Полигекс представляет собой связную фигуру на плоскости, составленную из конечного числа единичных правильных шестиугольников, примыкающих друг к другу по целым сторонам. Полигексы можно рассматривать как конечные подмножества шестиугольного паркета со связной внутренностью. Название полигекс было предложено Д. Кларнером [18]. Полигексы изучались в работах Гарднера [1-4], Голомба [5], Роадса [24], Майерса [22], Малеева $[6,21]$, Факуды $[14,15]$ и других авторов. Большой вклад в 
популяризацию математических задач, связанных с полигексом, внес Гарднер, который в своей рубрике “Математические игры” в журнале Scientific American опубликовал серию статей, обсуждающих эти проблемы, а затем включил их в соответствующие главы своих книг [1-4].

В настоящее время достаточно популярны многочисленные задачи, связанные с полигексами. В частности, различают свободные полигексы (когда фигуры, полученные поворотом или отражением, считаются одной и той же фигурой), односторонние полигексы (фигуры, полученные зеркальным отражением, читаются различными), фиксированные полигексы, различаемые при поворотах и т.д. Интерес к задачам перечисления полигексов связан с химией ароматических углеводородов [11, 12].

Отметим, что полигексы являются частным случаем полиформ, то есть плоских или пространственных геометрических фигур, получаемых путем соединения соседних фигур по целым ребрам или граням. Также интерес представляют полиформы с ячейками в виде правильных треугольников (полиамонды) и квадратов (полимино). Обзор многочисленных результатов и нерешенных проблем, связанных с перечислением полиформ конкретного типа, можно найти в работах [17-19, 23].

Перейдем к изучению разбиений на полигексы.

Определение. Разбиение называется решетчатым, если любую фигуру разбиения можно перевести в любую другую фигуру разбиения, при этом все разбиение переходит в себя.

Другими словами, разбиение на полигоны считается решетчатым, если существует группа трансляций плоскости, которая действует транзитивно на множестве полигексагональных ячеек. При этом существующее множество трансляций образует решетку. В случае полигексов легко видеть, что данная решетка является подрешеткой гексагональной решетки.

Без ограничения общности, можно считать, что все вершины полигекса являются точками целочисленной гексагональной решетки. Пусть $n$ площадь полигекса, то есть полигекс состоит из $n$ правильных шестиугольников, площади 1 каждый. Возникает задача подсчитать число решетчатых разбиений $t(n)$ плоскости на полигексы заданной площади.

Определение. Разбиение называется правильным, если для любых двух его фигур существует движение из группы симметрий этого разбиения, переводящее одну фигуру в другую и при этом все разбиение в себя.

Очевидно, что решетчатые разбиения являются частным случаем правильных разбиений. Правильные разбиения классифицируются по группам симметрий ячейки разбиения, которых всего насчитывается 17 (кристаллографические группы). Решетчатым разбиениям соответствует группа $p 1$, следующей по сложности идет группа $p 2$, порожденная решеткой и преобразованием центральной симметрии.

Мы будем рассматривать р2-разбиения плоскости на гомеоморфные диску полигексы. Также мы будем предполагать, что решетка периодов раз- 
биения является подрешеткой целочисленной гексагональной решетки.

Определение. Разбиение называется $p 2$-разбиением, если любую фигуру разбиения можно перевести в любую другую фигуру разбиения параллельным переносом или центральной симметрией, причем это преобразование переводит все разбиение в себя.

Два разбиения плоскости будем считать эквивалентными, если существует движение плоскости, переводящее одно разбиение в другое.

В настоящей статье мы докажем следующее утверждение.

Теорема. Для числа $t(n) p 2$-разбиений плоскости на полигексы заданной площади $n$, решетка периодов которых является подрешеткой гексагональной решетки, справедлива следующая оценка:

$$
C_{1} \cdot 2^{n} \leq t(n) \leq C_{2} \cdot 3,42^{n} \cdot n^{5} .
$$

Ранее аналогичные результаты были получены авторами в задачах подсчета числа решетчатых разбиений и $p 2$ - разбиений плоскости на полимино заданной площади $[8,9]$.

Доказательство:

Оценка снизу. Возьмем произвольную последовательность длины $n$ из нулей и единиц. По ней восстановим полигекс из $n+1$ шестиугольника единичной площади. 0 и 1 определяют способ стыковки соседних ячеек, а именно: 0 , если следующий шестиугольник смежен с предыдущим по нижнему горизонтальному ребру; 1 , если следующий шестиугольник смежен с предыдущим по боковому правому нижнему ребру. Итак, каждому такому коду будет поставлен в соответствие полигекс. Если для данного полигекса сначала сделать центральную симметрию в ребре крайнего шестиугольника этого полигекса, а потом сдвигать эту конструкцию уже из $2 n$ шестиугольников на векторы $(1 ;-1)$ и $(0 ; 2 n)$ гексагональной решетки, то мы получаем $p 2$-разбиения плоскости с решеткой периодов, порождаемой данными базисными векторами. Строение полигекса будет определяться цепочкой из 0 и 1 , и количество различных полигексов, определяемых этой последовательностью будет $2^{\text {n }}$. Некоторые из полученных разбиений могут совпадать, а именно: правильный шестиугольник имеет 6 осей симметрий и 6 поворотов, совмещающих шестиугольник с собой. Этим объясняется появление константы $\mathrm{C}_{1}$ в оценке снизу.

Оценка сверху. Вместо площади будем фиксировать периметр полигекса. Обозначим через $t^{\prime}(p)$ число $p 2$-разбиений плоскости на полигексы полупериметра $p$. Данное определение корректно, так как периметр любого полигекса четное число. Для $p 2$-разбиения существует критерий Конвея [25], устанавливающий при каких условиях полигекс задает $p 2$-разбиение, а именно:

Теорема: Полигекс порождает $p 2$-разбиение плоскости тогда и только тогда, когда граница полигекса может быть разбита на 6 частей $a b c d e f$ таких, что $a$ переходит в $d$ параллельным переносом, остальные части центрально- 
симметричны, причем некоторые части могут быть пустыми. При этом различным разбиениям границы полигекса соответствуют различные $p 2-$ разбиения плоскости на данный полигекс.

Известно, что число $m(l)$ самонепересекающихся блужданий длины $l$ на шестиугольной решетке не превосходит $C(\varepsilon)(\sqrt{2+\sqrt{2}}+\varepsilon)^{l}[13]$. Пусть $m_{c}(l)-$ число самонепересекащихся центрально-симметричных ломаных длины $l$. Такая ломаная определяется своей половиной, которая также является самонепересекающейся. Таким образом, справедлива оценка

$$
m_{c}(l)=\left\{\begin{array}{l}
m((l+1) / 2), \text { если } l \text { нечетно; } \\
m(l / 2), \text { если } l \text { четно }
\end{array}\right.
$$

Отсюда получаем, что для любого $\varepsilon>0$ :

$$
m_{c}(l) \leq m((l+1) / 2) \leq C^{\prime}(\varepsilon)(\sqrt{2+\sqrt{2}}+\varepsilon)^{l / 2} .
$$

Исходя из сказанного выше, для числа $t^{\prime}(p) p 2$-разбиений плоскости на равные полигексы полупериметра $p$ справедлива оценка:

$$
\begin{aligned}
& t^{\prime}(p) \leq \sum_{l_{a}+\frac{1}{2}\left(l_{b}+l_{c}+l_{e}+l_{f}\right)=p} m\left(l_{a}\right) m_{c}\left(l_{b}\right) m_{c}\left(l_{c}\right) m_{c}\left(l_{e}\right) m_{c}\left(l_{f}\right) \leq \\
& \leq C^{\prime}(\varepsilon) \sum_{l_{a}+\frac{1}{2}\left(l_{b}+l_{c}+l_{e}+l_{f}\right)=p}(\sqrt{2+\sqrt{2}}+\varepsilon)^{l_{a}}(\sqrt{2+\sqrt{2}}+\varepsilon)^{l_{b} / 2}(\sqrt{2+\sqrt{2}}+\varepsilon)^{l_{c} / 2}(\sqrt{2+\sqrt{2}}+\varepsilon)^{l_{e} / 2} . \\
& \cdot(\sqrt{2+\sqrt{2}}+\varepsilon)^{l_{f} / 2}=C^{\prime}(\varepsilon) \sum_{l_{a}+\frac{1}{2}\left(l_{b}+l_{c}+l_{e}+l_{f}\right)=p}(\sqrt{2+\sqrt{2}}+\varepsilon)^{l_{a}+\frac{1}{2}\left(l_{b}+l_{c}+l_{e}+l_{f}\right)}= \\
& =C^{\prime}(\varepsilon) \sum_{l_{a}+\frac{1}{2}\left(l_{b}+l_{c}+l_{e}+l_{f}\right)=p}(\sqrt{2+\sqrt{2}}+\varepsilon)^{p}=C^{\prime}(\varepsilon)(\sqrt{2+\sqrt{2}}+\varepsilon)^{p} \sum_{l_{a}+\frac{1}{2}\left(l_{b}+l_{c}+l_{e}+l_{f}\right)=p} 1 \leq \\
& \leq C^{\prime \prime}(\varepsilon)(\sqrt{2+\sqrt{2}}+\varepsilon)^{p} p^{4} \text {. }
\end{aligned}
$$

Последняя оценка связана с тем, что $\sum_{l_{a}+\frac{1}{2}\left(l_{b}+l_{c}+l_{e}+l_{f}\right)=p} 1$ является количеством решений линейного диофантового уравнения $l_{a}+\frac{1}{2}\left(l_{b}+l_{c}+l_{e}+l_{f}\right)=p$, их количество из [7] асимптотически с точностью до константы эквивалентно $p^{4}$.

Итак, мы получили, что число решетчатых разбиений плоскости на равные полигексы заданного полупериметра $p$ не превосходит

$$
t^{\prime}(p) \leq C^{\prime \prime}(\varepsilon)(\sqrt{2+\sqrt{2}}+\varepsilon)^{p} p^{4} .
$$

Перейдем к площади. Методом математической индукции легко получить, что периметр $P \leq 4 n+2$, тогда для полупериметра верно $p \leq 2 n+1$. Для получения верхней оценки числа решетчатых разбиений плоскости на полигексы заданной площади остается просуммировать предыдущую оценку по $p$ от 1 до $2 n+1$ : 


$$
t(n) \leq \sum_{p=1}^{2 n+1} t^{\prime}(p) \leq \sum_{p=1}^{2 n+1} C^{\prime \prime}(\varepsilon)(\sqrt{2+\sqrt{2}}+\varepsilon)^{p} p^{4} .
$$

Заменяя последнюю сумму на интеграл $\int_{0}^{2 n+1} C^{\prime \prime}(\varepsilon) x^{4}(\sqrt{2+\sqrt{2}}+\varepsilon)^{x} d x$ и учитывая, что

$$
\int x^{4} e^{\alpha x} d x=\frac{e^{\alpha x}}{\alpha^{5}}\left(\alpha^{4} x^{4}-4 \alpha^{3} x^{3}+12 \alpha^{2} x^{2}-24 \alpha x+12\right),
$$

получаем оценку

$$
t(n) \leq \sum_{p=1}^{2 n+1} C^{\prime \prime \prime}(\varepsilon)(\sqrt{2+\sqrt{2}}+\varepsilon)^{2 n}(2 n+1)^{4} .
$$

Ограничимся неравенством $\sqrt{2+\sqrt{2}}+\varepsilon \leq 1,85$, и, заменяя константу $C^{\prime \prime \prime}(\varepsilon)$ на $\bar{C}$, получаем оценку $t(n) \leq \bar{C}(2 n+1)^{5} 1,85^{2 n}$.

Занося все константы в константу $C_{2}$, имеем неравенство $t(n) \leq C_{2} n^{5} 3,42^{n}$.

Работа выполнена при поддержке РФФИ, гранты 17-02-00835-а и 17-42-330787.

\section{Список литературы}

1. Гарднер М. Путешествие во времени. М.: Мир, 1990. 341 с.

2. Гарднер М. Математические головоломки и развлечения. М.: Мир, 1999. 447 с.

3. Гарднер М. Математические досуги. М.: Мир, 1972. 496 с.

4. Гарднер М. Математические новеллы. М.: Мир, 1974. 456 с.

5. Голомб С. Полимино. М.: Мир, 1975. 207 с.

6. Малеев А.В., Шутов А.В. О числе трансляционных разбиений плоскости на полимино. Апатиты: Труды IX Всероссийской научной школы «Математические исследования в естественных науках», 2013. 101-106 с.

7. Нестеренко Ю.В., Галочкин А.И., Шидловский А.Б. Введение в теорию чисел. М.: Издательство Московского Университета, 1984. 147 с.

8. Шутов А.В., Коломейкина Е.В. Оценка числа решетчатых разбиений плоскости на центрально-симметричные полимино заданной площади. Яросл.: Моделирование и анализ информационных систем, 2014. 20, 148-157 с.

9. Шутов А.В., Коломейкина Е.В. Оценка числа $p 2$ - разбиений плоскости на полимино заданной площади. Тула: Чебышевский сб., 17:3, 2016. 204-214. 10. Brlek S., Frosini A., Rinaldi S., Vuillon L. Tilings by translation: enumeration by a rational language approach. The electronic journal of combinatorics, 13, 2006. 11. Dias J.R. A periodic Table for Polycyclic Aromatic Hidrocarbons. 1. Isomer Enumeration of Fused Polycyclic Aromatic Hidrocarbon. J. Chem. Inf. Comput. Sci., 22, 1982. 15-22.

12. Dias J.R. A periodic Table for Polycyclic Aromatic Hidrocarbons. 2. Polycyclic Aromatic Hidrocarbons Tetragonal, Pentagonal. J. Chem. Inf. Comput. Sci. 22, 1982. 15-22.

13. Duminil-Copin H., Simonov S. The connective constant of the honey comb lattice equals $\sqrt{2+\sqrt{2}}$. Annals of Mathematics. 175:3, 2012. 1653-1665.

14. Fukuda H., Mutoh N., Nakamura G., Schattschneider D. Enumeration of Polyominoes, Polyamonds and Polyhexes for Isohedral Tilings with Rotational Symmetry. Berlin: KyotoCGGT LNCS 4535 H. Ito et. al., 2008. 68-78. 
15. Fukuda H., Mutoh N., Nakamura G., Schattschneider D. A method to generate Polyominoes and Polyamonds for tilings with rotational symmetry. Berlin: Graphs ang Combinatirics, 23, 2007. 259-267.

16. Gardner M. Ch. 11. Polygexes and Polyaboloes. NY.: Mathematical Magic Show, 1978. 146-159.

17. Harary F. The cell growth problem and its attempted solutions. Leipzig: Beitrage zur Grathen theory, 1968. 49-60.

18. Klarner D. A cell growth problems. Cand. J. Math. 19, 1967. 851-863.

19. Klarner D.A., Rivest R.L. A procedure for improving the upper bound for the number of n-ominoes. Canad. J. Math., 25, 1973. 585-602.

20. Knop J.V., Szymanski Z., Trinajstie N. On the total number of polygexes. Match: Commun. Math. Chem., 16, 1984. 119-134.

21. Maleev A.V. Algorithm and computer-program search for variantsof polyhexes packing in plane. Crystallography Reports, 60, 2015. 986-992.

22. Myers J. Polyomino, polygex and polyamond tiling. http://www.srcf.ucam. org/jsm28/tiling/.

23. Read R.C. Contributions to the cell-growth problem. Canad. J. Math., 14, 1962. 1-20.

24. Rhoads G.C. Planar tilings by polyominoes, polyhexes and polyamonds. Journal of Computational and Applied Mathematics, 174, 2005. 329-353.

25. Schattschneider D. Will it tile? Try the Conway criterion! Math. Magazine, $53: 4,1980.224-233$.

\section{АЛГОРИТМЫ ПЕРЕБОРА ВАРИАНТОВ РЕШЁТЧАТЫХ И ПЕРИОДИЧЕСКИХ УПАКОВОК ТРЕХМЕРНЫХ ПОЛИКУБОВ}

Серавкин К.Г., Малеев А.В., Шутов А.В.

https://doi.org/10.31241/MIEN.2018.14.12

Владимирский государственный университет им. А.Г. и Н.Г. Столетовых, Владимир

\section{Аннотация}

Одной из важных задач кристаллографии и кристаллохимии является выявление особенностей молекулярных упаковок в кристаллическом состоянии. В связи с этим возникает, с одной стороны, проблема математического описания взаимного расположения молекул в кристалле по возможности меньшим числом параметров, с другой стороны, задача априорного предсказания возможных кристаллических структур данного химического соединения. Именно эти две задачи призван решать предложенный в [5] и обобщённый в [3] метод дискретного моделирования молекулярных упаковок в кристаллах (МДМ). Метод основан на замене молекул их дискретными моделями - поликубами - и расчёте всех возможных вариантов расположения этих поликубов в дискретном упаковочном пространстве.

\section{Summary}

One of the main problems of crystallography and crystal chemistry is to reveal the peculiarities of molecular packings in the crystal state. In this regard, there is, on the one hand, the problem of the mathematical description of the relative placement of the molecules in the crystal with less as possible parameters, on the other hand, the problem 